CARNETS DE Carnets de géographes

GÉOGRAPHES

$6 \mid 2013$

Géographie des faits religieux

\title{
Politiques de la vieillesse dans la région Pays de la
} Loire

\section{Mickaël Blanchet}

\section{(2) OpenEdition \\ Journals}

Édition électronique

URL : http://journals.openedition.org/cdg/868

DOI : $10.4000 /$ cdg. 868

ISSN : 2107-7266

Éditeur

UMR 245 - CESSMA

Référence électronique

Mickaël Blanchet, «Politiques de la vieillesse dans la région Pays de la Loire », Carnets de géographes [En ligne], 6 | 2013, mis en ligne le 01 septembre 2013, consulté le 24 septembre 2020. URL : http:// journals.openedition.org/cdg/868; DOI : https://doi.org/10.4000/cdg.868

\section{(c) $\oplus \Theta \Theta$}

La revue Carnets de géographes est mise à disposition selon les termes de la Licence Creative Commons Attribution - Pas d'Utilisation Commerciale - Pas de Modification 4.0 International. 


\section{POLITIQUES DE LA VIEILLESSE DANS LA REGION DES PAYS DE LA LOIRE}

\section{MICKAEL BLANCHET}

Dans un contexte de croissance du vieillissement de la population, cette thèse de géographie sociale s'est attachée, à l'échelle de la Région des Pays de la Loire, à évaluer les conséquences sociales et spatiales des politiques gérontologiques territorialisées.

Les recherches se sont déroulées en trois temps. Pour commencer, les bases de données démographiques, médicales, sociales et économiques relatives au vieillissement de la population et à l'offre de services et d'établissements gérontologiques ont été mobilisées afin de dresser un état des lieux du vieillissement régional. Ce travail a ensuite été suivi d'analyses multivariées débouchant sur une typologie régionale de la vulnérabilité des personnes âgées (figure 1). Ces opérations ont alors permis de sélectionner quatre départements et six intercommunalités d'étude propices à l'étude géographique des interactions entre les acteurs gérontologiques et les personnes âgées.

L'étude de ces interactions s'est centrée sur les acteurs gérontologiques qui évaluent et répondent aux besoins des personnes âgées. 127 entretiens semi-directifs, tous doublés, ont été nécessaires à la réalisation de cette étape. Ces entretiens distinguent l'échelle institutionnelle (Conseils Généraux, administrations d'État, conseils de l'ordre des médecins et caisses de retraite : 16 entretiens) de l'échelle locale où 111 d'acteurs gérontologiques locaux (médecins généralistes, infirmières libérales, services de soins infirmiers à domicile, maisons de retraites, services d'aide à domicile...) ont été sondés.

À la suite d'un long travail d'analyse et de cartographie, il a été possible de ressortir les pratiques spatiales de ces mêmes institutions et acteurs.

Enfin, la dernière phase de recherche a eu pour but d'évaluer le rapport local des personnes âgées à l'offre de services et d'établissements gérontologiques. Menées dans les intercommunalités d'observation, ces recherches se sont appuyées sur des sources institutionnelles relatives aux activités gérontologiques (allocataires de l'allocation personnalisée autonomie, minimum vieillesse, taux d'équipement, consommations médicales, activités des services...) ainsi que sur la diffusion d'un questionnaire auprès de 900 personnes âgées portant sur les pratiques, le rapport local à l'offre et les solidarités familiales en situation de besoin. 
Figure 1 : Typologie territoriale de la vulnérabilité des personnes âgées dans la région Pays de la Loire

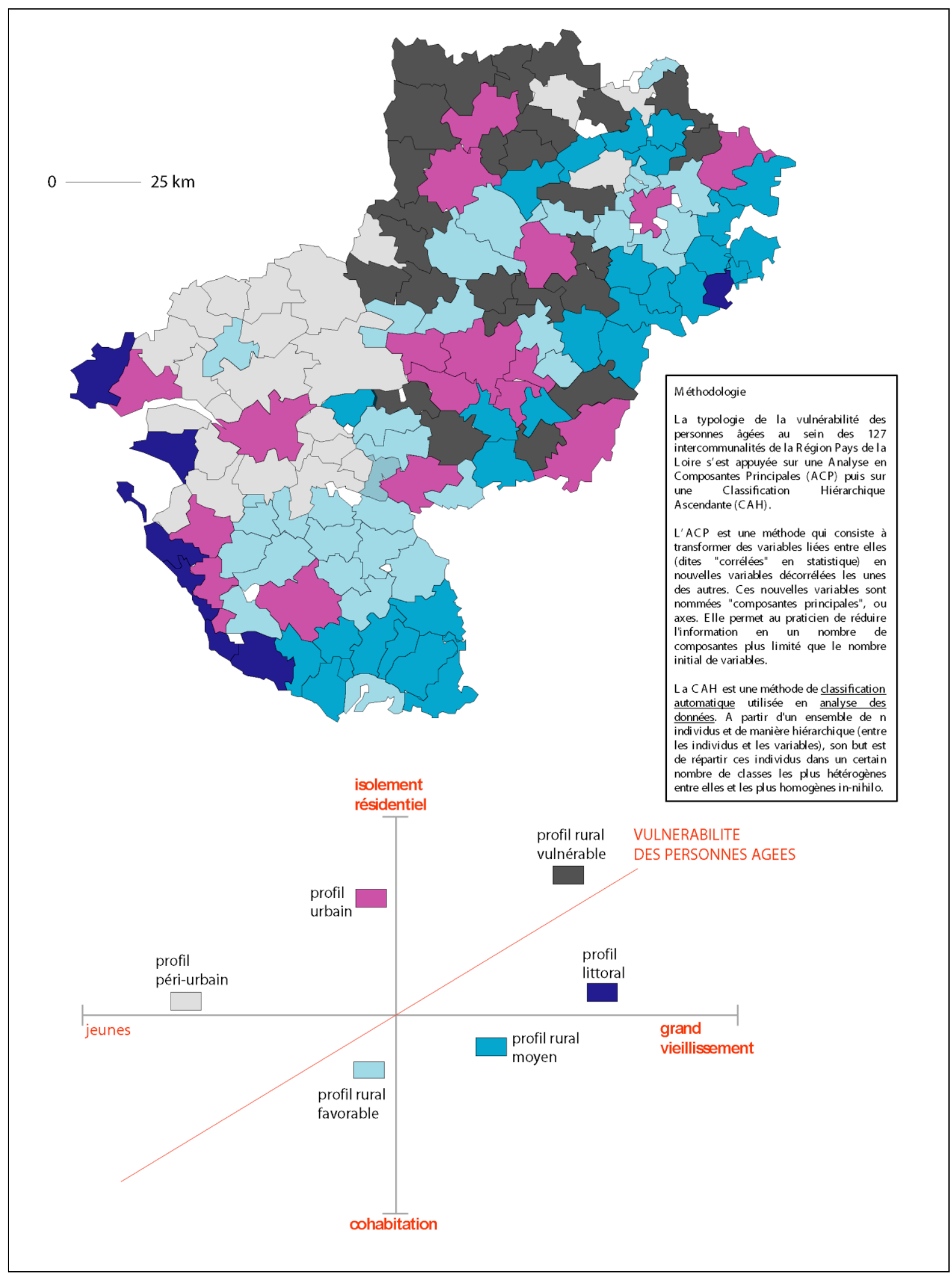

Mickaël BLANCHET, Université d'Angers, 2011

Sources : recensements 1999 et 2009, enquête revenus 2008 -Insee Pays de la Loire 
Pour répondre aux besoins des personnes âgées, les pouvoirs publics, notamment les Conseils Généraux et les Agences Régionales de Santé, se sont appuyés sur le territoire pour mener à bien leurs réponses. Néanmoins, le développement sectoriel et l'ouverture aux normes économiques des politiques gérontologiques se sont traduits par une dispersion de l'action gérontologique et par de nouvelles inégalités de répartition de l'offre. En effet, le passage d'une logique d'établissement à une logique de besoin a eu pour conséquence de multiplier et de superposer sans cohérence ni concertation les cadres de régulation de l'action gérontologique et d'accroitre les inégalités de l'offre.

Résultat, les villes concentrent les activités les plus spécialisées et soumises à la concurrence et les campagnes affichent une moindre diversité et spécialisation de l'offre où le binôme aide à domicile en milieu rural- maisons de retraites médicalisées structure l'action gérontologique locale.

Dans ce contexte, les recherches sur les régulations entre les besoins des personnes âgées, l'offre locale de services et d'établissements et les solidarités familiales mettent en avant trois formes de régulation :

- une première forme assistantielle dans les zones rurales les plus profondes où les pouvoirs publics et les services sociaux comblent les distances familiales.

- une seconde forme en milieu rural, où les solidarités familiales s'inscrivent en complément d'un niveau moyen de l'offre locale.

- une troisième forme dans les zones les plus denses et les plus solvables, où malgré leurs proximités, les familles tendent à se désengager en faveur d'un développement élevé et multiple de l'offre de services et d'établissements.

Figure 2 : Régulations locales entre les personnes âgées, les aidants informels et les professionnels

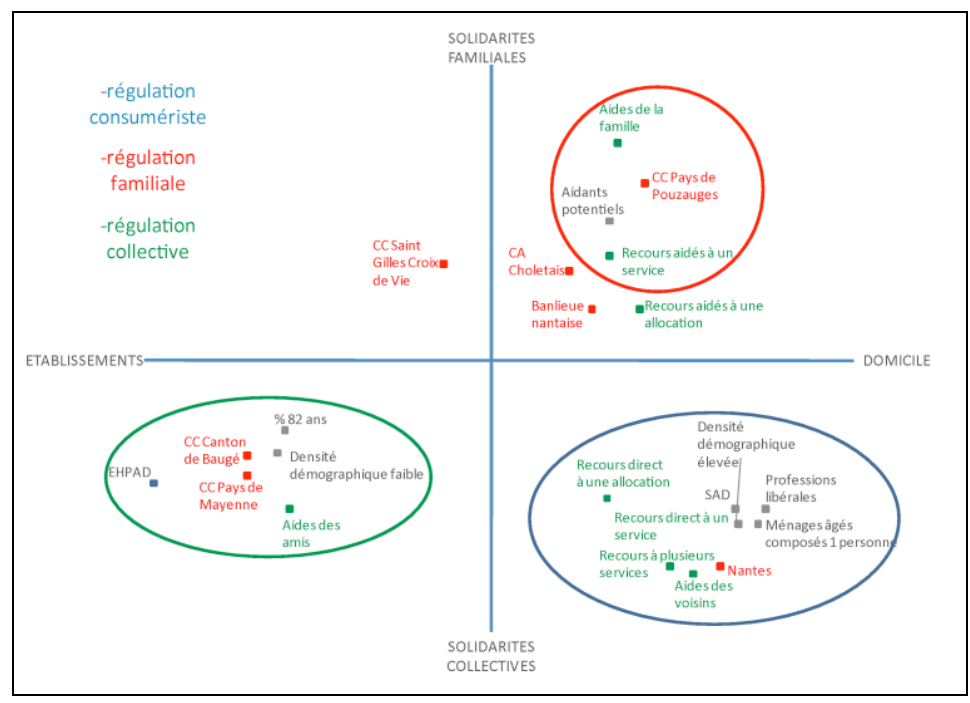

Source : recherches personnelles, Mickaël Blanchet, 2011 - méthode utilisée : Analyse en Composantes Principales 
Il ressort de ces régulations l'adaptation des personnes âgées aux inégalités locales de niveau et de diversité de l'offre gérontologique. Dans ce jeu, les populations âgées aisées (les moins prédisposées aux situations de dépendance et d'isolement) ont les capacités géographiques, économiques et sociales à se référer aux pratiques d'indépendance et de choix. En revanche, les populations âgées les moins aisées et les plus fragiles situées en périphérie rurale et urbaine ne présentent ni les prédispositions géographiques, ni les capacités économiques pour se démarquer des productions normatives qui découlent des politiques de la vieillesse.

L'objectif de la thèse était, à partir d'un espace précis, d'analyser l'organisation spatiale des politiques de la vieillesse pour mieux faire ressortir ses conséquences géographiques. En ce sens, la thèse montre une gestion spatiale des problématiques gérontologiques entre espaces dotés de capacités économiques et professionnelles et espaces moins riches et moins structurés professionnellement. Cette gestion territorialisée s'avère au final inverse aux objectifs affichés par les pouvoirs publics, à savoir l'adaptation aux besoins des personnes âgées. Devant la croissance à venir de personnes âgées dépendantes et de retraités en situation de précarité/pauvreté, il sera intéressant de suivre dans la région Pays de la Loire mais aussi dans d'autres espaces, l'évolution et les conséquences de ce mode gestion par poche, par segment des politiques la vieillesse.

\section{Fiche informative}

Discipline

Géographie

Directeur

Christian Pihet

Université

Université d'Angers

Membres du Jury de thèse, soutenue le 7 avril 2011

- M. Yves JEAN, Professeur de Géographie, Université de Poitiers, rapporteur

- M. Jean-Marc MACE, Professeur de Géographie, CNAM Paris, rapporteur

- M. François MADORE, Professeur de Géographie, Université de Nantes, examinateur

- M. Henry NOGUES, Professeur Emérite d'Economie, Université de Nantes, examinateur

- M. Christian PIHET, Professeur de Géographie, Université d'Angers Directeur de Thèse

- M. Jean SOUMAGNE, Professeur de Géographie, Université d'Angers, Président du Jury

Situation professionnelle actuelle

Post-doctorat de géographie sur la "Prise en charge territoriale de la maladie d'Alzheimer " - Université d'Angers - EHESP

Courriel de l'auteur :

mickaelblanchetfr@gmail.com 